\section{Herramientas digitales para la obtención de registros, posicionamiento y articulación virtual de modelos}

\section{Digital tools for obtaining records, positioning and virtual articulation of casts}

\section{Resumen}

La siguiente revisión bibliográfica busca describir las alternativas digitales disponibles para la obtención de registros, posicionamiento y articulación virtual de modelos junto con ejemplificar algunos softwares que permitan la implementación de estas tecnologías en su trabajo diario. Se realizó una búsqueda de literatura en las bases de datos: Pubmed, LILACS, SciELO y Cochrane Library. Finalmente, luego de ser filtrados los resultados, se utilizaron un total de 36 publicaciones. Existen diversas herramientas disponibles actualmente para digitalizar una gran cantidad de técnicas que solo hasta hace algunos años eran realizadas manualmente por un operador. Algunas de ellas, facilitan una inmersión paulatina al mundo virtual del odontólogo novato ya que carecen de altos costos y recursos. Se sugiere que los odontólogos implementen estas tecnologías en su trabajo habitual según cada caso particular.

Palabras clave: Consultorios odontológicos; Prostodoncia; Flujo de trabajo; Técnica de Colado Dental (fuente: DeCS BIREME).

\begin{abstract}
The following literature review seeks to bring together the digital alternatives available for obtaining records, positioning and virtual articulation of models together with exemplifying some softwares to allow the implementation of these technologies in their daily workflow. A literature search was carried out in the following databases: Pubmed, LILACS, SciELO and Cochrane Library. Finally, after the results were filtered, a total of 36 publications were used. There are various tools currently available to digitize a large number of techniques that were only performed manually by an operator until a few years ago. Some of them facilitate a gradual immersion into the virtual world of the novice dentist since they lack high costs and resources. It is suggested that dentists implement these technologies into daily dental practice according to each particular case.
\end{abstract}

Keywords: Dental Offices; Prosthodontics; Workflow; Dental casting technique (source: MeSH NLM).

\section{Artículo de Revisión}

\author{
Paula Díaz Díaz ${ }^{\text {1,a, Javiera Muñoz Solís }}{ }^{2, a}$, David Contreras \\ Diez de Medina ${ }^{3, b}$ \\ ${ }^{1}$ Universidad Mayor, Facultad de Odontología, Santiago, \\ Chile. \\ 2 Pontificia Universidad Católica de Chile, Facultad de \\ Odontología, Santiago, Chile. \\ ${ }^{3}$ Universidad Andrés Bello, Facultad de Odontología, \\ Santiago, Chile. \\ a Diplomado en Ortopedia y Ortodoncia en Anomalías \\ Dentomaxilares. \\ ${ }^{\mathrm{b}}$ Diplomado en Crecimiento y Desarrollo Craneofacial \\ aplicado a la Ortodoncia Interceptiva.
}

\section{Correspondencia: \\ Paula Díaz Díaz: pfdiazd@gmail.com \\ Bueras 359, OF 609-610, Rancagua, Chile \\ ORCID: 0000-0003-4232-3222}

\section{Coautores:}

Javiera Muñoz Solís: javmunozs@udd.cl

ORCID: 0000-0002-8609-134X

David Contreras Diez de Medina: dcontrerasdiezdemedina@gmail.com

ORCID: 0000-0002-4176-0060

\section{Editora:}

Maria Eugenia Guerrero Acevedo

Universidad Nacional Mayor de San Marcos, Perú

Conflicto de interés: los autores declaran no tener conflicto de interés.

\section{Fuente de financiamiento: autofinanciado.}

\section{Recibido: 29/09/20}

Aceptado: 09/11/20

Publicado: 15/02/21 


\section{Introducción}

En el quehacer odontológico habitual existen numerosos procedimientos indispensables para un adecuado tratamiento integral, dentro de los cuales se encuentra la toma de registros y montaje de modelos en el articulador. Sin embargo, estos procedimientos son considerados engorrosos y desagradables desde el punto de vista de los pacientes, odontólogos y laboratoristas, quienes deben dedicar tiempo, espacio y materiales adicionales para su elaboración. A esto, se suma que existen dificultades inherentes a los procedimientos técnicos y los materiales utilizados, tales como la deformación del registro de mordida, la estabilidad del articulador, el correcto posicionamiento de los modelos, la contracción del yeso, la destreza que debe tener el tratante, entre otros; que obstaculizan la precisión y reducen la fiabilidad del uso de esta técnica ${ }^{1,2}$.

Los articuladores son instrumentos mecánicos que representan la mandíbula, el maxilar y la articulación temporomandibular permitiendo a los profesionales simular algunos o todos sus movimientos sin la necesidad de contar con el paciente de manera presencial ${ }^{1}$. Sin embargo, se ven limitados para representar los efectos de la resistencia de los tejidos blandos, el patrón de movimiento de masticación guiado por los músculos ${ }^{3} \mathrm{y}$ las condiciones dinámicas reales de la articulación ${ }^{4}$.

Con los avances de la odontología moderna se ha concebido una nueva categoría en la clasificación, los articuladores clase $\mathrm{V}$ o virtuales, los cuales simulan las condiciones reales del paciente con más precisión y menos errores, permitiendo disminuir el tiempo de sillón aumentando la comodidad del paciente y eficiencia de tratamiento ${ }^{5,6}$. Además, se facilita el trabajo para el laboratorista dental quien utiliza esta herramienta como soporte para la confección asistida por computador de restauraciones indirectas ${ }^{7}$. Las múltiples novedades tecnológicas y en definitiva, el advenimiento de la digitalización en odontología han vuelto más fácil, preciso y rápido el quehacer de los profesionales en la actualidad ${ }^{3}$. Sin embargo, resulta incongruente notar que, a pesar de que la evolución de los articuladores digitales se inició hace más de 20 años con el articulador de Szentpetery en $1999^{2-4}$, en la actualidad esta herramienta no sea de amplio acceso o uso, principalmente por su alto costo ${ }^{8}$.

La siguiente revisión bibliográfica tiene como finalidad describir las alternativas digitales disponibles para la obtención de registros, posicionamiento y articulación virtual de modelos para que los odontólogos se informen y posteriormente puedan implementar estas tecnologías en su clínica diaria.

\section{Revisión de la literatura actual}

En el presente artículo de revisión se realizó una búsqueda de literatura en las siguientes bases de datos: Pubmed, LILACS, SciELO, Cochrane Library y en la revista "The journal of prosthetics dentistry", utilizando los descriptores: odontología digital, flujo de trabajo digital, articulador virtual, articulador digital, escáner intraoral y técnica de montaje de modelos, en inglés y español. Se filtraron los resultados por antigüedad de 10 ańos (2010-2020) y se incluyeron revisiones sistemáticas, revisiones de literatura, reporte de técnicas y textos de acceso completo obteniéndose 714 resultados. También se realizó una búsqueda ampliada de artículos potencialmente no encontrados mediante el método manual en las listas de referencias según autor. Luego se procedió a leer los resúmenes y se excluyeron aquellos estudios que no tuvieron relación con el tema de investigación y duplicados. De tal modo, se seleccionaron 36 documentos que abordaban en sus contenidos la información atingente al presente estudio.

\section{Flujo de trabajo odontológico digital y análogo para la obtención de registros, montaje y articulación de modelos virtuales}

Para el estudio de un caso; el flujo de trabajo, comienza con la toma de registros, luego se realiza el montaje del modelo superior y finalmente el montaje del modelo inferior (Figura 1). En el método análogo se inicia con la toma de impresiones de los modelos y registro

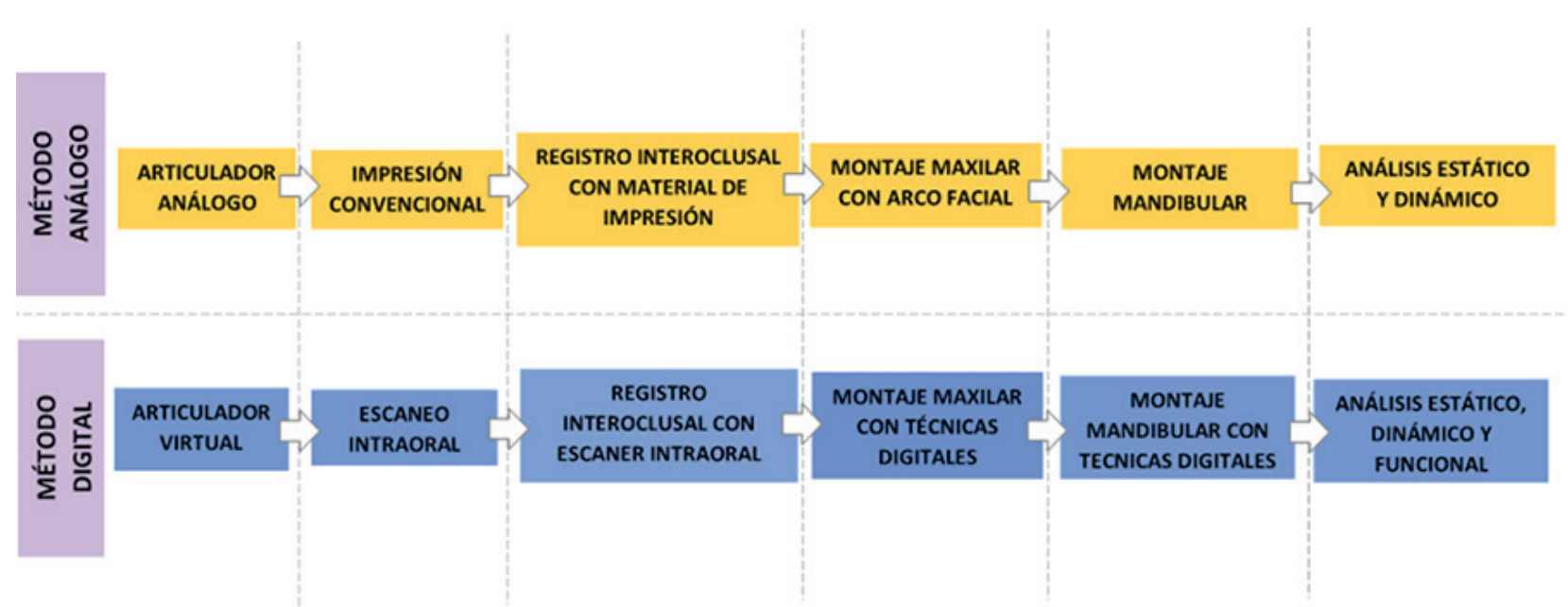

Figura 1. Diagrama de la sistemática para la toma de registros y montaje de modelos, comparando el método análogo y su equivalente digital 
interoclusal, luego se obtiene la posición maxilar mediante el arco facial, y finaliza el proceso con el montaje mandibular. En tanto, el método digital utiliza escáneres intraorales para la toma de impresiones y registro interoclusal, luego para obtener la relación del maxilar con respecto al cráneo se pueden utilizar distintas metodologías que pueden incluir desde el uso del escáner facial como el de un arco facial virtual y, finalmente, el montaje mandibular se realiza a través de softwares que poseen algoritmos que permiten coincidir las intercuspidaciones con el registro interoclusal digital ${ }^{9}$.

\section{Evolución y desarrollo del articulador}

Los articuladores son instrumentos mecánicos que representan la mandíbula, el maxilar y la articulación temporomandibular permitiendo a los profesionales simular algunos o todos sus movimientos mandibulares con los dientes en contacto sin la necesidad de contar con el paciente de manera presencial ${ }^{9}$. Se clasifican en no ajustables (clase I), semi ajustables (clase II y III) y totalmente ajustables (clase IV). El origen de cada uno de ellos proviene de diversas escuelas oclusionistas que buscaban describir los fenómenos involucrados en la reproducción del movimiento mandibular y al mismo tiempo idear instrumentos simples que puedan ser utilizados en la clínica ${ }^{10}$. A pesar de la evolución que han tenido estos instrumentos, este escenario mecánico tan diferente del entorno biológico de la vida real plantea una serie de dificultades, ya que siguen los márgenes de las estructuras que conforman la articulación mecánica las cuales permanecen invariables en el tiempo y que no pueden simular movimientos de dinámica real mandibular confiables ${ }^{11,12}$. Es así, como surge el articulador digital o clase $\mathrm{V}$ que ofrece la posibilidad de reducir significativamente las limitaciones de los articuladores mecánicos ${ }^{13}$.

La evolución de los articuladores digitales se inicia en el año 1999 con el desarrollo del articulador de Szentpetery, matemáticamente ajustable. Luego en el 2000, Kordass y Gaertner desarrollaron el primer articulador completamente ajustable que utiliza un rastreador de movimiento mandibular ${ }^{1,3,12}$. En 2009, Solabarrieta et al. ${ }^{14}$ se propuso desarrollar un articulador virtual mediante el uso de ingeniería inversa basándose en los articuladores manuales Hanau H2 y el Ivoclar Stratos 2000. Durante la primera década del 2000, los esfuerzos se centraron en desarrollar softwares con articuladores virtuales integrados basados en articuladores manuales existentes, de tal manera que; los odontólogos y técnicos dentales sean quienes decidan cuál es el articulador ideal para la simulación de cada paciente ${ }^{3,4,12}$.

\section{Clasificación de los articuladores digitales}

Actualmente, los articuladores virtuales se pueden dividir en:

a. Matemáticamente ajustables. Se basan en una simulación matemática para representar los movimientos mandibulares actuando como un articulador con valores promedio. Debido a que el montaje en estos articuladores se considera arbitrario, muchos estudios han intentado estandarizar la relación existente entre el eje horizontal transversal mediante el uso de puntos de referencia anatómicos. Kois et al. ${ }^{15}$ midió la distancia entre un eje transversal horizontal arbitrario -cuyo centro se encontraba en los cóndilos mandibulares y los rebordes alveolares, en la cara lingual de los centrales inferiores generando un triángulo, en donde la media de sus lados fue de 100,12 mm. Este hallazgo fue consistente con los estudios previos realizados por Bonwill con la postulación de su triángulo equilátero, y la esfera de Monson ${ }^{15,16}$. Por lo tanto, los articuladores digitales matemáticamente ajustables basan el montaje de los modelos en un triángulo equilátero de $100 \mathrm{~mm}$ de lado, con vértices en los cóndilos y en la cara lingual de los incisivos centrales inferiores ${ }^{15}$ (Figura 2).
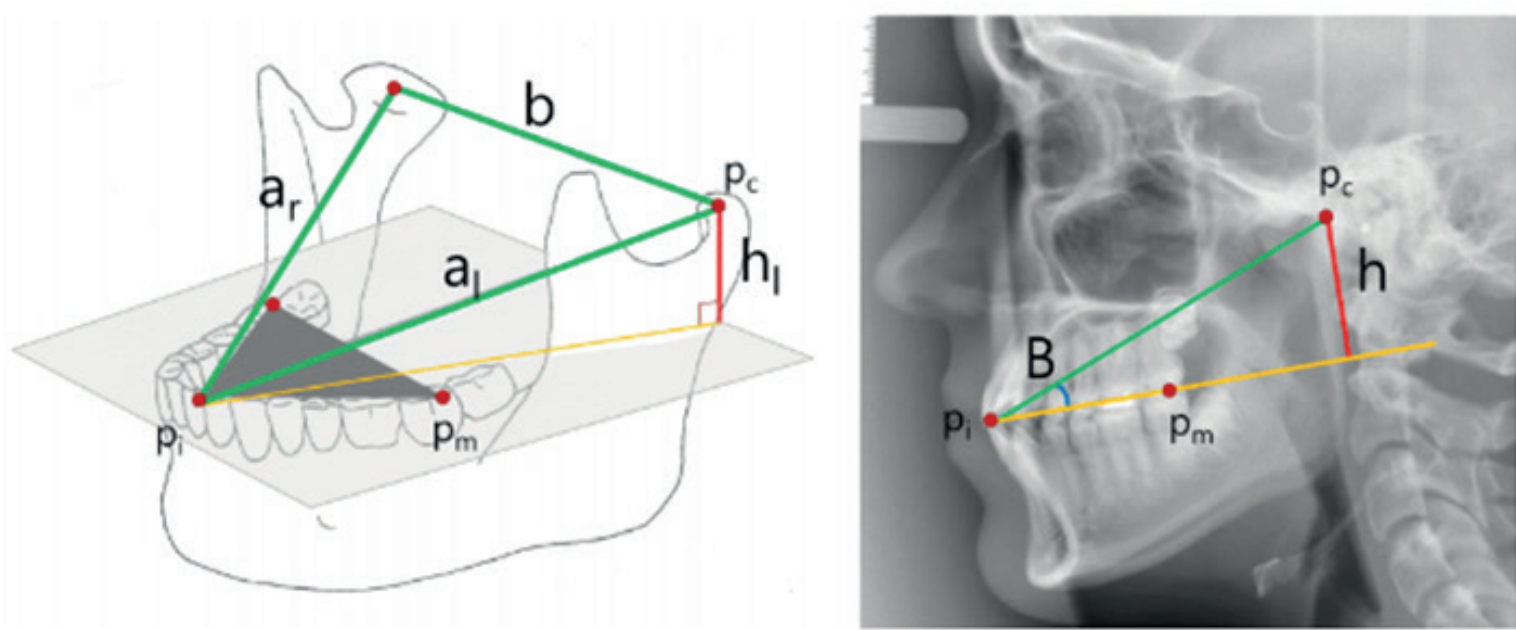

Figura 2. Puntos de referencia anatómicos para el articulador virtual (Redibujando desde Ohm 1982; Maggetti 2015)

${ }^{14}$. Triángulo de Bonwill (triángulo verde); plano oclusal (plano gris y línea amarilla); "a" corresponde al brazo derecho e izquierdo del triángulo de Bonwill; "b" a la base del triángulo; " $h$ " a la altura; "B" al ángulo de Balkwill; "pi" al punto medio del borde incisal; "pm" a la punta de la cúspide distobucal del segundo molar y "pc" al centro del cóndilo 
b. Completamente ajustables. Corresponden a articuladores digitales a los que se le pueden importar registros que reproducen exactamente los movimientos de la mandíbula utilizando un sistema electrónico de registro denominado jaw motion tracking system (JMT) ${ }^{1}$. Este sistema fue desarrollado por Kordass y Gaertner por primera vez en el año $2000^{1-4,12}$. El JMT es un sistema basado en sensores ultrasónicos u ópticos, que rastrean la mandíbula del paciente durante sus movimientos funcionales. Se compone principalmente de un software, un arco facial, adaptadores oclusales y múltiples transmisores a nivel de los incisivos mandibulares y receptores en el arco facial que detectan los movimientos de traslación y rotación de la mandíbula. Además, ciertas marcas permiten la integración completa con sistemas de registro intraoral y tomografía computarizada volumétrica (TCV) ${ }^{4}$.

\section{Métodos de obtención de registros}

El diseño de un articulador digital y la reproducción de la articulación dental a grandes rasgos se realiza mediante ingeniería inversa ${ }^{1,12}$. Inicialmente, los articuladores virtuales se obtenían de modelos mecánicos que eran escaneados mediante un scanner de mesa, de tal manera que, se clasificaban igual que los mecánicos. Con el tiempo, han sido íntegramente diseñados de forma virtual. Los avances de hoy en día permiten articular los modelos con la representación anatómica exacta de la articulación temporomandibular del paciente al utilizar las imágenes de una TCV y agregar datos como la relación intermaxilar estática, relación cráneo mandibular dinámica, registro de actividad muscular mediante una electromiografía u otro método que capte los desplazamientos de la mandíbula ${ }^{1,12}$. Es por ello que la adquisición de estos registros y la exactitud y fiabilidad de ellos se convierte en un elemento tan fundamental ${ }^{17}$.

La adquisición de los datos necesarios para su utilización en un flujo digital odontológico puede obtenerse mediante: un escaneo intraoral directamente de la boca del paciente -digitalización directa-; el escaneo extraoral de una impresión o modelo de yeso -digitalización indirecta- y el escaneo de un articulador con los modelos montados -digitalización indirecta-. Ambos tipos de digitalizaciones tienen como producto un archivo STL (stereolitography) renderizado del registro digitalizado ${ }^{17}$.

a. Digitalización indirecta (Realizada mediante un escáner de mesa). Esta herramienta emplea un rayo láser no destructivo y varias cámaras digitales para reproducir imágenes de alta resolución de las superficies. Las impresiones, modelos o registros de mordida se colocan en el interior de una plataforma, en donde la cámara se gira e inclina automáticamente durante el escaneo generando un modelo STL. Los modelos de yeso, impresiones y el registro de mordida pueden ser descartados, eliminando la necesidad de almacenamiento ${ }^{7}$.

b. Digitalización directa (Realizada mediante escáner intraoral). Son dispositivos constituidos principalmente por una estación móvil de trabajo que recolecta datos, un monitor para visualizar escaneos u archivos digitales y una cámara intraoral que reúne datos directamente de la boca del paciente. Para reunir puntos de datos de superficie, la energía de la luz láser o la luz blanca se proyecta desde la cámara intraoral sobre un objeto y se refleja de nuevo en un sensor dentro de la cámara. Según los algoritmos, se toman decenas o cientos de miles de mediciones por pulgada, lo que da como resultado una representación tridimensional de la forma del objeto. A su vez, también permite registrar la posición de mordida del paciente mediante escaneos en oclusión, permitiendo registrar arcadas en máxima intercuspidación (MIC) o en relación céntrica $(\mathrm{RC})$ posterior a su manipulación ${ }^{18}$.

\section{Softwares}

Para visualizar los registros STL obtenidos de la digitalización, estos se deben importar a softwares de diseño asistido por computador ("computer aided design") -de ahora en adelante CAD. Existen una variedad de opciones de softwares CAD en el mercado, pero por fines prácticos sólo serán abordados softwares de diseño para uso clínico y que posean módulos de articulación, los cuales serán divididos en programas de licencia adquirida y programas de acceso gratuito u Open Source ${ }^{19}$.

Los softwares CAD de licencia adquirida con módulos de articulación digital incorporados más utilizados en el mercado son: 3Shape (3Shape A/S), Exocad DentalCAD (Exocad GmbH), Cerec (Sirona), Zirkonzhan Modellier (Zirkonzhan) y Ceramill Artex (Artex Cr) (Figura 3); los cuales poseen herramientas para simular movimientos mandibulares que pueden ser ajustables al paciente usando valores numéricos para representar la inclinación condilar, el ángulo de Bennet, dimensión vertical, etc. Además, otorgan la posibilidad de articular los modelos mediante la importación de registros en MIC o RC y algunos permiten la incorporación de archivos TCV en el montaje de modelos ${ }^{20}$. Sin embargo, el elevado costo de estas licencias ${ }^{20}$, junto con la necesidad de capacitación ${ }^{20,21}$, y las limitaciones de homologación de archivos entre los mismos softwares, introdujo la aparición en el mercado de softwares Open Source, los cuales corresponden a programas de diseño -no necesariamente odontológicos- de acceso gratuito. Dentro de esta categoría encontramos a BlueSky Plan (V4.1.9, Blue Sky Bio, Grayslake IL) y Meshmixer (Meshmixer, Autodesk, San Rafael, CA). Bluesky Plan comenzó como una compañía de implantes, a medida que el uso de TCV creció vieron la necesidad de creación de un software de visualización gratuita y de uso universal. Posee un módulo de articulación dentaria que solo requiere modelos virtuales con registro de mordida en céntrica, MIC o ambos para generar la articulación. Una de sus mayores virtudes es que permite incorporar un registro TCV al montaje de modelos. Por otro lado, Meshmixer no es un software de uso dental, sino que es un programa de edición de archivos STL cuyo diseño es básico y amigable; y posee diversas herramientas 
que pueden aplicarse al flujo digital odontológico. Este programa no posee un módulo de articulación dentaria en sí, pero los modelos pueden articularse mediante la creación de un pivote unido a un triángulo cuyas aristas midan $100 \mathrm{~mm}$ cada una, emulando de esta forma al triángulo de Bonwill como se observa en la Figura 4. El registro interdental es reconocido por el programa, permitiendo que los modelos y sus relaciones interdentarias se puedan ubicar de forma correcta ${ }^{7}$.

Es importante recalcar que, en todos los softwares mencionados anteriormente, la articulación de los modelos se realiza de forma arbitraria. Los modelos digitales se comportan de manera similar a un objeto flotante en el espacio ya que no se encuentran precisamente orientados en las coordenadas X, Y, Z después de ser escaneados digitalmente, lo que en definitiva hace difícil el poder identificar líneas medias, inclinación del plano oclusal y por, sobre todo, la posición del maxilar superior sin los puntos de referencia adecuados ${ }^{20}$.

\section{Métodos de posicionamiento del modelo superior en articuladores digitales}

Después de la obtención de los registros digitales surge la interrogante “Cómo debo posicionar en el espacio estos modelos?". Si bien para la realización de restauraciones indirectas digitalmente no es esencial el posicionamiento correcto del maxilar superior de acuerdo con algún plano de referencia, es necesario y fundamental si la digitalización del paciente es con fines diagnósticos, ortodóncicos, quirúrgicos o por fines interdisciplinarios ${ }^{22}$.

Actualmente, los articuladores matemáticamente ajustables se encuentran integrados a un software de diseño que permite configurar arbitrariamente sus parámetros. Es por lo anterior que durante el último tiempo se han propuesto diferentes métodos para transferir la posición del maxilar superior a los articuladores virtuales de manera fidedigna, siendo alguna de las técnicas que destacan: El uso de un arco facial análogo y escaneo del articulador con modelos montados; fotografías extraorales estandarizadas; posicionamiento mediante platina; uso de un escáner facial; creación de un arco facial virtual y la integración del TCV para el montaje de modelos $8,20,22-27$.

Es importante mencionar que, hasta el momento, ninguna técnica ha demostrado su eficiencia, fiabilidad y practicidad por sobre las otras ${ }^{8,20,22-27}$, sino más bien se clasifican como "Reporte de una técnica"; las cuales no serán abordadas en el presente artículo.
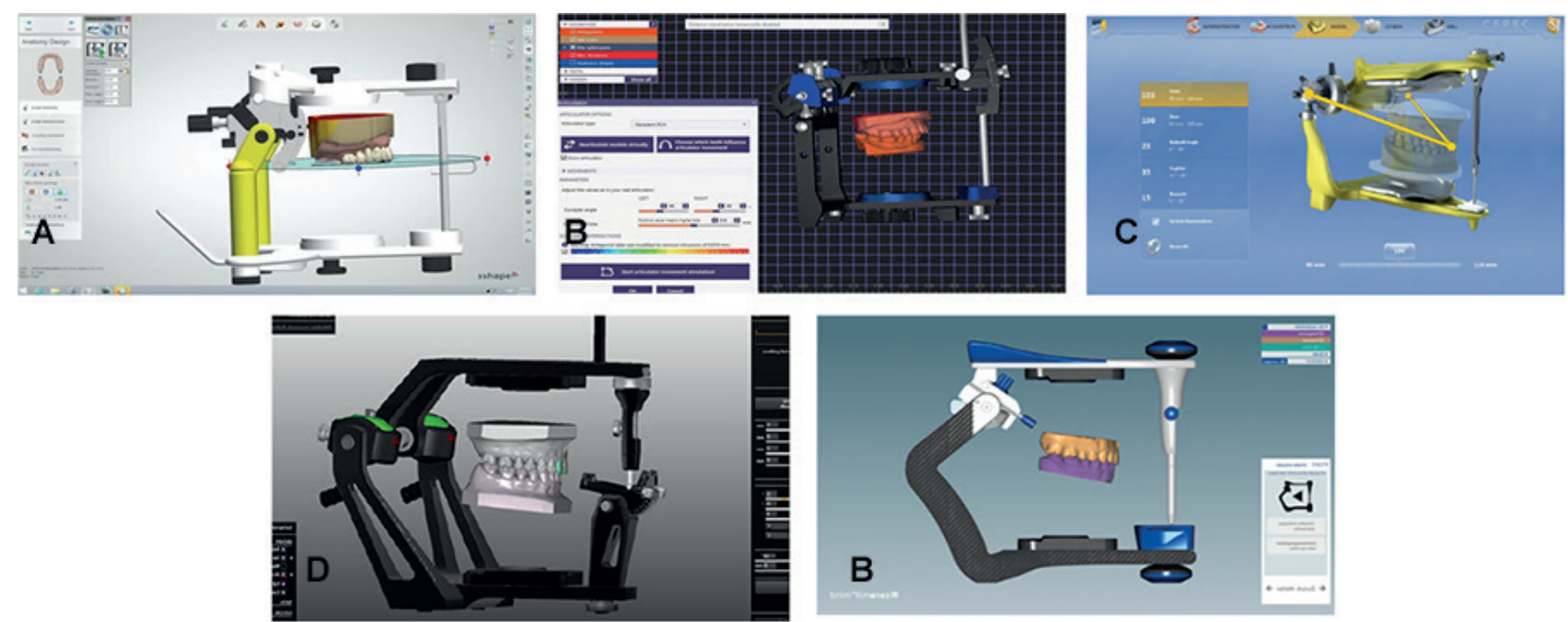

Figura 3. Tipos de Softwares CAD de licencia adquirida con módulo de articulación virtual integrado. En A el módulo de 3Shape, B módulo de Exocad, C módulo de Cerec, D módulo de Zirkonzhan y E módulo de Ceramill. Imágenes obtenidas de los sitios web de los fabricantes
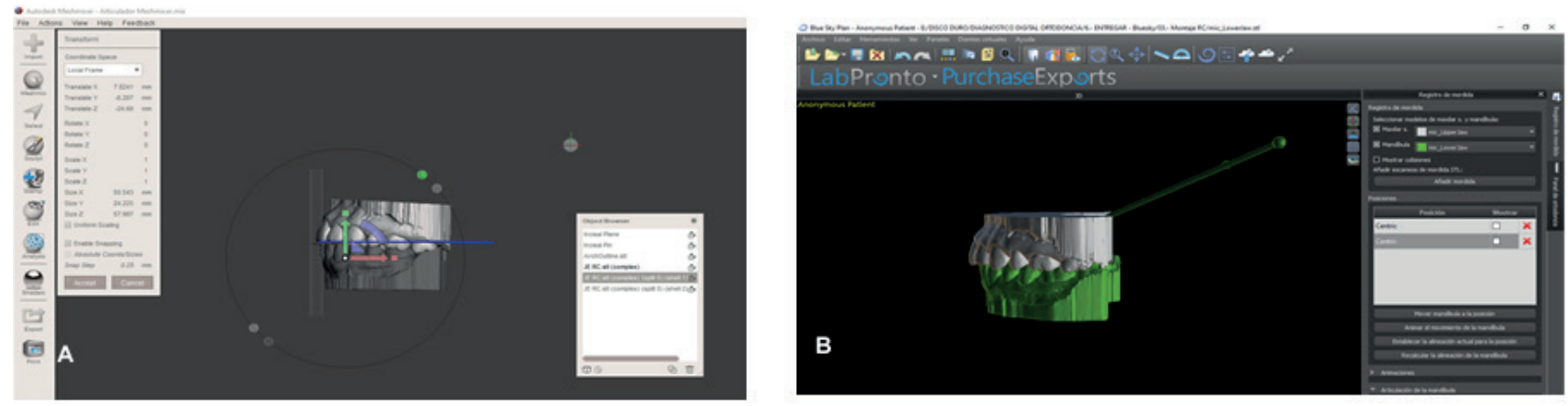

Figura 4. Tipos de Softwares Open Source con posibilidad de articular modelos. En A se observa Meshmixer, En B se observa el módulo de articulador virtual de Blue Sky Plan. Imágenes obtenidas durante la planificación de un caso usando dichos softwares 


\section{Análisis funcional mediante sistemas de rastreo mandibular (Jaw Movement Tracking Systems)}

Corresponden a sistemas que permiten registrar el movimiento tridimensional en tiempo real de la mandíbula, también conocido como rastreo de movimiento mandibular (JMT). Existen diferentes sistemas en el mercado, los que se basan principalmente en dos principios: ultrasónicos y ópticos. Dentro de los ultrasónicos encontramos a SICAT Function ${ }^{\mathrm{TM}}$ y al Jaw Motion Analyzer (JMA) ${ }^{\mathrm{TM}}$. El JMA ${ }^{\mathrm{TM}}$ es el predecesor del SICAT Function $^{\mathrm{TM}}$, por lo que el principio y la interfaz de operación son muy similares ${ }^{28}$, ambos dispositivos capturan el movimiento por un conjunto de emisores de ultrasonido que se encuentran adheridos a las superficies vestibulares de los dientes mandibulares usando una plantilla personalizada ${ }^{28}$.

Dentro de los sistemas ópticos, encontramos el Planmeca $4 \mathrm{D}^{\mathrm{TM}}$ Jaw Motion y el Modjaw ${ }^{\mathrm{TM}}$. El principio de funcionamiento de ambos se basa en la triangulación óptica mediante cámaras posicionadas en el arco facial y la emisión de LED infrarrojos en la mandíbula. El Planmeca $4 \mathrm{D}^{\mathrm{TM}}$ Jaw Motion es un procesador de operación que tiene un dispositivo de posicionamiento para la toma de TCV y su uso se reduce sólo para diagnosticar desórdenes temporomandibulares ya que su registro no se puede integrar a un software CAD ${ }^{27}$. Por otra parte, $\mathrm{Modjaw}^{\mathrm{TM}}$ utiliza un lápiz de posicionamiento óptico para conectar el escáner bucal, por lo que puede combinarse con un escáner intraoral sin necesidad de tomar una TCV lo que significa una reducción en la dosis de radiación recibida por el paciente ${ }^{28,29}$.

El uso de registros JMT no solo funcionan como complemento diagnóstico durante el análisis del paciente, sino que también ayudan al clínico durante el desarrollo de una rehabilitación, disminuyendo en gran medida el tiempo necesario en el sillón para ajustes funcionales y minimizan el tiempo necesario para que el paciente se acostumbre a su nueva restauración ${ }^{28,29}$.

\section{Implementación del flujo digital en la clínica}

$\mathrm{La}$ integración transversal de tecnologías $\mathrm{CAD}$ en nuestro país y a nivel latinoamericano no tiene valores estimados. En recientes estudios se menciona que en el Reino Unido el $56 \%$ de los odontólogos no usa o no está interesado en implementar algún tipo de tecnología CAD/CAM en su práctica, y en Suiza, sólo el $23 \%$ de los clínicos utilizan esta tecnología en sus consultas ${ }^{30}$. Si bien estos números pueden considerarse desalentadores, una de las premisas importantes de este estudio fue que se observó que, a menor edad del odontólogo, existía una mayor posibilidad de implementación tecnológica en su práctica clínica ${ }^{30,31}$, lo cual contrasta con el grado de integración digital en las mallas curriculares de pregrado, las que en su mayoría carecen de educación digital ${ }^{32}$. Hoy en día la tecnologización de la clínica se relaciona más a una motivación personal que a una necesidad real, y es en este ímpetu en el que recae la creación de técnicas híbridas o digitalmente indirectas que permiten adentrarse de manera paulatina en este flujo de trabajo. La técnica de digitalización indirecta consiste en la implementación de métodos análogos para la obtención de registros durante el flujo de trabajo digital ${ }^{33}$, en donde, por ejemplo, los modelos de yeso y los registros de mordida en formato físico pueden ser escaneados por un scanner de mesa y de esta forma adquirir los modelos y sus relaciones de manera digital ${ }^{34}$. Siendo de gran beneficio, ya que permiten externalizar procedimientos si no se cuenta con el equipamiento o conocimiento necesario para realizarlo ${ }^{34}$. En la Figura 5, se grafica a nivel general los pasos necesarios si un clínico desea implementar un flujo de trabajo digital en su práctica diaria; cada técnica es mencionada con su paralelo digital y análogo en la misma fila.

\section{Discusión}

El uso de herramientas que simulan la realidad clínica del paciente sin necesariamente contar con su presencia ha sido implementado por los odontólogos desde hace años, siendo el articulador el elemento caracterizador. Estos instrumentos han evolucionado en el tiempo, y hace 20 años que se dispone de sus versiones digitales. El principio de funcionamiento de un articulador virtual se basa en las teorías tradicionales de la oclusión ${ }^{35}$, ya que utiliza el triángulo de Bonwill como método de posicionamiento arbitrario de modelos digitales, el cual -recordemos- es el mismo principio que utilizan ciertos articuladores análogos semiajustables. Sin embargo, resulta llamativo notar que, a pesar de que la virtualización de los articuladores lleva más de 2 décadas, en la actualidad esta herramienta no sea de amplio acceso ni uso ${ }^{32}$.

Las virtudes del mundo digital benefician tanto al tratante, al paciente y al laboratorista. Para el dentista, las ventajas del escaneo digital influyen en un diagnóstico mejorado y planificación del tratamiento, mayor aceptación de casos, envío más rápido de registros a laboratorios, menos repeticiones, tiempo reducido en el consultorio, estandarización de procedimientos de oficina, requisitos de almacenamiento reducidos ${ }^{18}$, retorno de laboratorio más rápido, mayor precisión, flujo de trabajo mejorado, menor gasto de inventario y tiempos de tratamiento reducidos. Para el paciente incluyen una presentación mejorada del caso y una mejor experiencia en la atención dental, con más comodidad y menos ansiedad, disminución de tiempo en sillón y una fabricación más fácil de aparatos perdidos o rotos, así como un tiempo de tratamiento potencialmente reducido ${ }^{18}$. En el laboratorio, los modelos digitales dan opciones flexibles para el diseño y la fabricación de una amplia gama de restauraciones dentales, implantes, modelos de estudio, aparatos como soportes indirectos personalizados, prótesis fijas unitarias, incrustaciones, expansores, alineadores, retenedores, etc ${ }^{7}$.

Por otra parte, también es importante mencionar que, en el ámbito de investigación, el número de ensayos clínicos que evalúen flujos de trabajo digitales completos es bajo, y los que existen generalmente estudian y comparan su utilización para confección de restauraciones indirectas. 


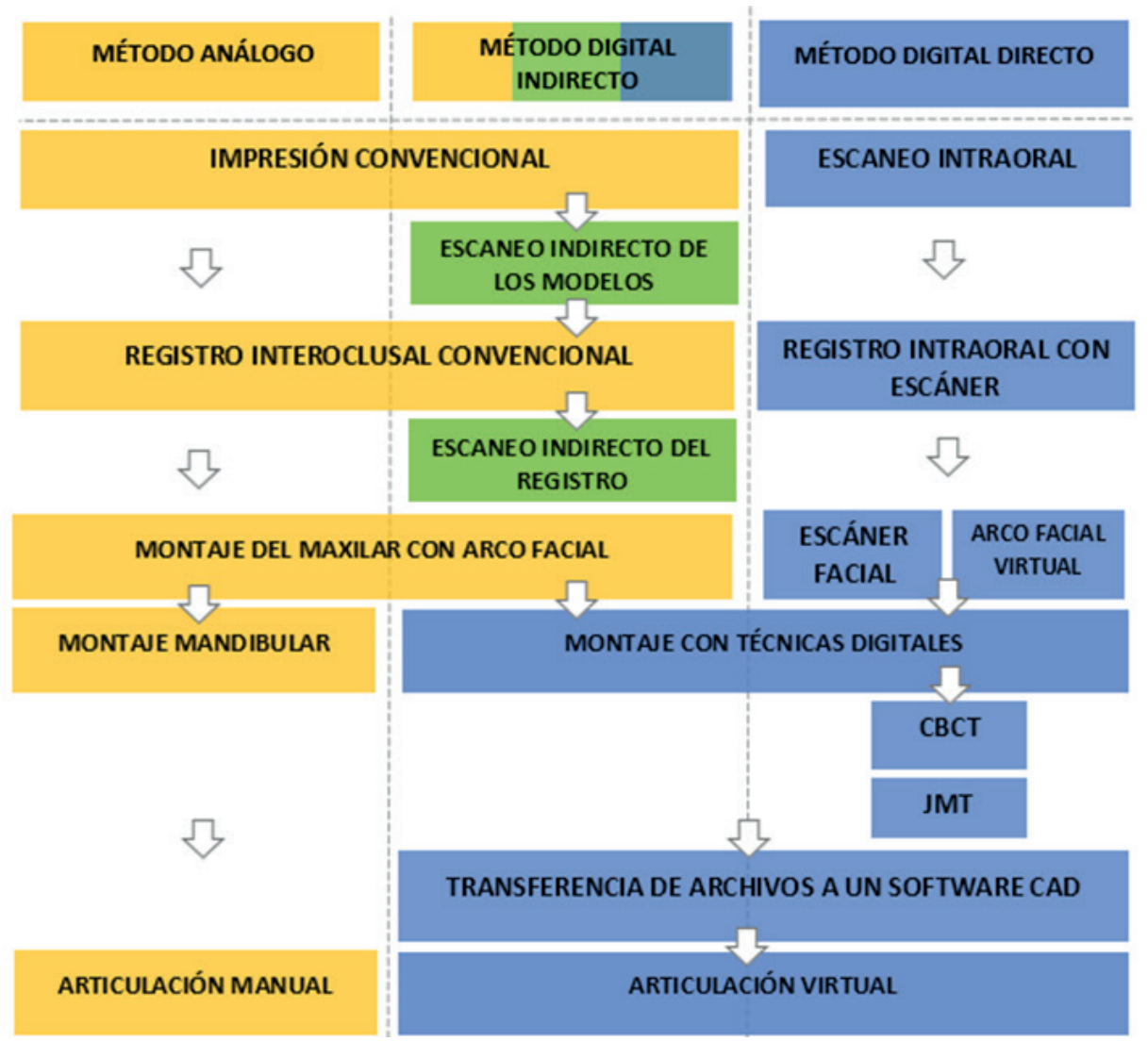

Figura 5. Diagrama que ejemplifica la implementación digital en la práctica con su equivalente análogo y digital directo e indirecto. En la columna central, se introduce el método digital indirecto, punto intermedio entre la técnica análoga y la completamente digital

Al parecer, la investigación con ensayos de alta calidad parece ser más lenta que el progreso industrial de las aplicaciones digitales disponibles ${ }^{36}$. La investigación futura con ensayos clínicos aleatorios bien diseñados, incluida la observación de seguimiento, es absolutamente necesaria en el campo del procesamiento digital completo para poder implementar estas tecnologías con base científica de respaldo. Esto también sucede con las múltiples opciones existentes para posicionar modelos sobre articuladores digitales; si bien las técnicas son diversas, los estudios no presentan parámetros estandarizados que permitan corroborar su exactitud y precisión ${ }^{8,20,22-27}$.

La gran disrupción que se plantea en este artículo es que existen alternativas de transición que permiten una mayor accesibilidad a las tecnologías tridimensionales, sin un costo económico mayor, con la posibilidad de externalizar procesos a laboratorios si no se cuenta con equipos digitalizadores. En este sentido, los softwares Open Source que ofrecen herramientas para el diagnóstico y planificación de casos sin suscripción a una licencia ${ }^{22,33}$, permiten ser un puente entre softwares de licencia CAD y el usuario principiante. Esto proporciona una introducción paulatina del odontólogo a la era digital. Los módulos de articulación de los softwares de diseño asistidos por computador (CAD) al ser de uso global poseen una interfase amigable para el usuario, lo que permite analizar y generar planes de tratamientos virtuales de forma más directa y menos engorrosa. Además, en recientes estudios de este año, Úry et al. ${ }^{8}$, indican que la precisión del espacio dental virtual utilizando métodos indirectos es confiable para el análisis oclusal virtual en la práctica clínica.

Si bien muchas virtudes pueden considerarse a la hora de digitalizar la práctica clínica, a futuro la gran piedra de tope ya no será la accesibilidad a estos softwares y equipos, sino más bien la curva de aprendizaje necesaria para su utilización. En este sentido, sería de gran utilidad la introducción de estas herramientas desde la formación de pregrado a un nivel multidisciplinario; en donde no solamente se introduzca a los alumnos el flujo digital mediante softwares CAD asociados a licencias, sino que sería interesante el presentarles softwares Open Source para que ellos dispongan de las herramientas necesarias y pensamiento crítico si deciden digitalizar su práctica clínica como dentistas titulados ${ }^{32}$. Es imperioso que los odontólogos implementen las tecnologías digitales en su flujo de trabajo diario ya que los beneficios son múltiples. Las diversas alternativas permiten que cada odontólogo, -en base a su habilidad y herramientas disponibles-, decida qué alternativa utilizar para cada caso particular. Finalmente, resulta imprescindible que el desarrollo de las tecnologías vaya de la mano con su implementación en la práctica clínica e investigación científica. 


\section{Conclusiones}

Existen diversas herramientas virtuales disponibles actualmente para registrar, posicionar y articular modelos de estudios. Entre estas, algunas incluyen pasos del sistema análogo, como las del método indirecto. Se sugiere que cada odontólogo en base a sus recursos decida qué método implementar; debido a que actualmente no hay evidencia concluyente que haya demostrado mayor eficiencia, fiabilidad y practicidad por sobre las otras.

\section{Referencias bibliográficas}

1. Luthra RP, Gupta R, Kumar N, Mehta S SR. Virtual articulators in prosthetic dentistry: A review. Adv Med Dent Scie Res. 2015;3(4):117-21.

2. Kordass B, Gärtner C, Söhnel A, Bisler A, Voss G, Bockholt $U$, et al. The virtual articulator in dentistry: concept and development. Dent Clin North Am. 2002;46(3):493-506. DOI: 10.1016/s00118532(02)00006-x.

3. Singh N, Dandekeri S, Shenoy K B V. Digital Articulators: A Promising Technology of the Future. Int J Dent Med Res. 2014;1(2):98-102.

4. Harasymiw B. Virtual Articulators in Prosthetic Dentistry: a Review. Can Slavon Pap. 2011;53(2-4):401-20.

5. Su T, Sun J. Comparison of repeatability between intraoral digital scanner and extraoral digital scanner: An in-vitro study. J Prosthodont Res. 2015;59(4):236-42. DOI: 10.1016/j.jpor.2015.06.002

6. Patzelt SBM, Lamprinos C, Stampf S, Att W. The time efficiency of intraoral scanners: an in vitro comparative study. J Am Dent Assoc. 2014;145(6):542-51. DOI: 10.14219/jada.2014.23

7. Taneva E, Kusnoto B, Evan, CA. 3D Scanning, Imaging, and Printing in Orthodontics. Issues in Contemp. orthod.: InTech, 2015; 147-88. DOI: 10.5772/60010

8. Úry E, Fornai C, Weber GW. Accuracy of transferring analog dental casts to a virtual articulator. J Prosthet Dent. 2020;123(2):305-13. DOI: $10.1016 /$ j.prosdent.2018.12.019

9. Son K, Lee KB. Effect of Tooth Types on the Accuracy of Dental 3D Scanners: An In Vitro Study. Materials (Basel). 2020;9;13(7):1744. DOI: 10.3390/ma13071744

10. Keith J. The Glossary of Prosthodontic Terms. 9th ed. J Prosthet Dent; Elsevier. 2017.

11. Manns A, Biotti J. Manuel Práctico de Oclusión Dentaria. 2da Ed, Amolca, 2008.

12. Koralakunte PR, Aljanakh M. The role of virtual articulator in prosthetic and restorative dentistry. J Clin Diagnostic Res. 2014;8(7):25-9. DOI: 10.7860/ JCDR/2014/8929.4648

13. Maestre-Ferrín L, Romero-Millán J, Peñarrocha-Oltra D, Peñarrocha-Diago M. Virtual articulator for the analysis of dental occlusion: an update. Med Oral Patol Oral Cir Bucal. 2012;17(1):160-3. DOI: 10.4317/medoral.17147

14. Solaberrieta E, Etxaniz O, Minguez R, Muniozguren J, Arias A. Design of a virtual articulator for the simulation and analysis of mandibular movements in dental
CAD/CAM. Compet Des - Proc 19th CIRP Des Conf. 2014;323-30

15. Kois JC, Kois DE, Chaiyabutr Y. Occlusal errors generated at the maxillary incisal edge position related to discrepancies in the arbitrary horizontal axis location and to the thickness of the interocclusal record. J Prosthet Dent. 2013;110(5):414-9. DOI: 10.1016/j.prosdent.2013.06.005

16. Ohm E, Silness J. The size of the Balkwill angle and the height of the Bonwill triangle. J Oral Rehabil. 1982;9(4):301-6. DOI: 10.1111/j.1365-2842.1982. tb01019.x

17. Ellakwa A, Elnajar S, Littlefair D, Sara G. A Novel Methodology to Validate the Accuracy of Extraoral Dental Scanners and Digital Articulation Systems. Eur J Prosthodont Restor Dent. 2018;26. DOI: 10.1922/EJPRD_01747Ellakwa10

18. Baheti MJ, Soni UN, Gharat NV, Mahagaonkar P KR and DS. Intra-oral Scanners: A New Eye in Dentistry. Austin J Orthopade Rheumatol. 2015;2(3):247-369.

19. Talmazov G, Bencharit S, Waldrop T, Ammoun R. Accuracy of Implant Placement Position Using Non-Dental Open-Source Software: An In Vitro Study. J Prosthodont. 2020;4. DOI: 10.1111/jopr.13208

20. Brenes C, Jurgutis L BC. Digital face-bow transfer technique using the dentofacial analyzer for dental esthetics and 2-D, 3-D smile design: A clinical report. J Oral Sci Rehabil. 2018;4(2):22-30.

21. Alghazzawi TF. Advancements in CAD/CAM technology: Options for practical implementation. J Prosthodont Res. 2016;60(2):72-84. DOI: 10.1016/j. jpor.2016.01.003

22. Lepidi L, Chen Z, Ravida A, Lan T, Wang HL, Li J. A Full-Digital Technique to Mount a Maxillary Arch Scan on a Virtual Articulator. J Prosthodont. 2019;28(3):335-8. DOI: 10.1111 /jopr.13023

23. Petre A, Drafta S, Stefanescu C, Oancea L. Virtual facebow technique using standardized background images. J Prosthet Dent. 2019;121(5):724-8.

24. Granata S, Giberti L, Vigolo P, Stellini E, Di Fiore A. Incorporating a facial scanner into the digital workflow: A dental technique. J Prosthet Dent. 2020;123(6):781785.

25. Lam WYH, Hsung RTC, Choi WWS, Luk HWK, Pow EHN. A 2-part facebow for CAD-CAM dentistry. J Prosthet Dent. 2016;116(6):843-7.

26. Solaberrieta E, Mínguez R, Barrenetxea L, Etxaniz O. Direct transfer of the position of digitized casts to a virtual articulator. J Prosthet Dent. 2013;109(6):411-4.

27. Solaberrieta E, Garmendia A, Minguez R, Brizuela A, Pradies G. Virtual facebow technique. J Prosthet Dent. 2015;114(6):751-5.

28. Chen H, Lee C. 2019 IDS Jaw Movement Tracing Systems - CEREC Digest [Internet]. CEREC Digest. 2019 [citado 1 Nov 2020]. Disponible en: https://www.cerecdigest.net/2019/06/24/2019-ids-jaw-movement-tracing-systems/ 
29. Madhavan S, Dhanraj M, Jain A. Methods of recording mandibular movements - A review. Drug Invent Today. 2018;10:1254-9.

30. Mühlemann S, Sandrini G, Ioannidis A, Jung RE, Hämmerle CHF. The use of digital technologies in dental practices in Switzerland: a cross-sectional survey. Swiss Dent J. 2019;129(9):700-7.

31. Van der Zande MM, Gorter RC, Bruers JJM, Aartman IHA, Wismeijer D. Dentists' opinions on using digital technologies in dental practice. Community Dent Oral Epidemiol. 2018;46(2):143-53. DOI: 10.1111/ cdoe. 12345

32. Zitzmann NU, Matthisson L, Ohla H, Joda T. Digital Undergraduate Education in Dentistry: A Systematic Review. Int J Environ Res Public Health. 2020;17(9):3269. DOI: $10.3390 /$ ijerph17093269

33. Lepidi L, Galli M, Grammatica A, Joda T, Wang H-L, Li J. Indirect Digital Workflow for Virtual Cross-Mounting of Fixed Implant-Supported Prostheses to Create a 3D Virtual Patient. J Prosthodont Off J Am Coll Prosthodont. 2020. DOI: 10.1111/jopr.13247
34. Vecsei B, Joós-Kovács G, Borbely J, Hermann P. Comparison of the accuracy of direct and indirect three-dimensional digitizing processes for CAD/CAM systems - An in vitro study. J Prosthodont Res. 2016;1:61. DOI: 10.1016/j.jpor.2016.07.001

35. Hong S-J, Noh K. Setting the sagittal condylar inclination on a virtual articulator by using a facial and intraoral scan of the protrusive interocclusal position: A dental technique. J Prosthet Dent. 2020. DOI: 10.1016/j.prosdent.2020.01.031

36. Kay T Oen, Analia Veitz-Keenan, Silvia Spivakovsky, Y Jo Wong, Eman Bakarman JY. CAD/CAM versus traditional indirect methods in the fabrication of inlays, onlays, and crowns. Cochrane Database Syst Rev. 2014;4(4). DOI: $10.1002 / 14651858 . C D 011063$ 
